\title{
Generalized Riccati equations for 1-D magnetotelluric impedances over anisotropic conductors Part I: Plane wave field model
}

\author{
Světlana Kováčiková and Josef Pek \\ Geophysical Institute, Acad. Sci. Czech Rep., Boční II/1401, CZ-14131 Prague 4-Spořilov, Czech Republic
}

(Received December 11, 2000; Revised September 17, 2001; Accepted September 18, 2001)

\begin{abstract}
In the 1-D magnetotelluric theory, a Riccati equation governs the depth variation of the impedance, or admittance, for a given distribution of the electrical conductivity. This equation can be used to compute the surface magnetotelluric functions for generally piecewise continuous conductivity profiles. In case of a simple layered medium, it provides the classical formulae for recalculating recursively the impedances between the individual layer boundaries. We present an extended version of the Riccati differential equations for generally anisotropic 1-D structures for the case of a plane wave incident field. Relation between the standard matrix propagation procedure for a layered medium and the Riccati equation approach, as a limiting case of the former, is demonstrated. In the anisotropic case, all elements of the $2 \times 2$ impedance tensor are present and, consequently, a system of four coupled Riccati equations has to be considered. Standard methods for the numerical solution of systems of ordinary differential equations are applied to the Riccati system, which gives an efficient alternative to the current matrix propagation procedures for the numerical evaluation of 1-D magnetotelluric impedances in anisotropic media. As an application, a synthetic study on the influence of a depth-variable regional strike on magnetotelluric decomposition results is presented, with the variable strike simulated by a variable anisotropy within the 1-D section.
\end{abstract}

\section{Introduction}

In a 1-D magnetotelluric (MT) model, with a generally piecewise continuous conductivity distribution $\sigma(z)$, the depth dependence of the impedance $Z(z, \omega)=E_{x} / H_{y}=$ $-E_{y} / H_{x}$, or admittance $Y(z, \omega)=Z^{-1}(z, \omega)$, is governed by a simple Riccati equation

$$
\begin{aligned}
& \frac{\partial Z(z, \omega)}{\partial z}-\sigma(z) Z^{2}(z, \omega)=i \omega \mu_{0}, \\
& \frac{\partial Y(z, \omega)}{\partial z}+i \omega \mu_{0} Y^{2}(z, \omega)=-\sigma(z),
\end{aligned}
$$

where $\omega$ is the circular frequency of the time-harmonic electromagnetic field, with the time factor $\exp (-i \omega t)$ considered, and $\mu_{0}$ is the free-space permeability (e.g., Dmitriev and Berdichevsky, 1979). Equations (1) are an elementary consequence of Maxwell's equations, written in a form considering the 1-D symmetry of the model. At a $z$ where $\sigma(z)$ suffers a discontinuity, (1) is completed by the condition of continuity of $Z(z, \omega)$, or $Y(z, \omega)$, which is again an elementary consequence of the basic continuity conditions for the field components through layer boundaries.

There is a close relation between (1) and the well known recurrent formulae commonly used for the impedance propagation through homogeneous layers of a stratified medium. In a homogeneous layer with the thickness $h$ and conductivity $\sigma$, the impedances on the top and bottom interfaces of

Copy right (C) The Society of Geomagnetism and Earth, Planetary and Space Sciences (SGEPSS); The Seismological Society of Japan; The Volcanological Society of Japan; The Geodetic Society of Japan; The Japanese Society for Planetary Sciences. the layer are related via

$$
\begin{aligned}
Z_{\mathrm{bot}}(\omega) & =-\frac{k}{\sigma} \tanh \left\{k h-\tanh ^{-1}\left[\frac{\sigma}{k} Z_{\mathrm{top}}(\omega)\right]\right\} \\
& =\frac{Z_{\mathrm{top}}(\omega)-k \sigma^{-1} \tanh k h}{1-\sigma k^{-1} Z_{\mathrm{top}}(\omega) \tanh k h}
\end{aligned}
$$

with $k=\sqrt{-i \omega \mu_{0} \sigma}$. By evaluating the limit of this relation when $h$ approaches zero, and by using $\tanh k h \approx k h$ if $h \rightarrow 0$, we immediately obtain (1). In this respect, Eqs. (1) can be considered a direct generalization of the impedance formulae for discrete homogeneous layers to media with generally piecewise continuous distribution of the electrical conductivity. Of course, the reverse is true as well, i.e., the above propagation formula for the impedances in a homogeneous layer can be proved to be a solution of the Riccati impedance equation (1) with $\sigma(z)=\sigma=$ const.

From the practical point of view, evaluating MT impedances by solving the Riccati equation (1) has several advantages. First, generally continuous and piecewise continuous conductivity distributions $\sigma(z)$ can be immediately considered. For a few simple conductivity functions, analytical solutions of the Riccati equation can be found. In those cases, valuable information could be obtained as to the sensitivity of the MT curves with respect to smooth transitions in the conductivity distribution (e.g., Singh and Kant, 1995). Second, the numerical solution of the Riccati equation is extremely simple, and allows us carrying out MT simulations immediately, without dealing with sometimes rather tedious matrix propagation algebra. We need only to know the starting impedance, which is usually the elementary halfspace impedance in the basement, and can proceed in simple 
steps through the model towards its surface. Third, the impedance is a much more regular function of depth than the field components themselves, which are, in a layer of a finite thickness, composed of a down-going and an up-going wave, both with an exponential depth dependence. This, numerically insecure, exponential dependence is eliminated if the impedance is regarded.

One-dimensional MT models with anisotropic layers have been analyzed from various points of view in a lot of papers during the last thirty years (e.g., O'Brien and Morrison, 1967; Reddy and Rankin, 1971; Loewenthal and Landisman, 1973; Abramovici, 1974; Dekker and Hastie, 1980). Except (Abramovici, 1974), all those publications consider layered structures. The earlier papers by O'Brien and Morrison (1967) and Reddy and Rankin (1971) are based on the matrix propagation method applied to the field components in the anisotropic layered medium, with the impedance tensor being a terminal product of the field computations. In the anisotropic case, the matrix algebra is much more involved as compared to the isotropic media. Moreover, two wave modes arise in each anisotropic layer, with possibly largely different wave numbers, which can be a source of further numerical inconveniences.

The more recent of the above mentioned articles use mostly the matrix propagation technique as a theoretical tool only, and derive more stable impedance propagation formulae for anisotropic layered structures. These already mark the way to an extension based on the Riccati equation approach to anisotropic media, with all its essential attributes of regularity and straightforwardness of the solution.

An approach based on the idea of using first-order differential equations to propagate the MT impedances through a 1-D anisotropic model was first suggested by Abramovici (1974). He derived a coupled set of six first-order linear differential equations for the depth derivatives of secondorder sub-determinants constructed from a field components matrix, which easily lead to the impedance elements in an anisotropic medium. Those equations are not completely closed with respect to the impedances in that they still explicitly contain the individual field components. Abramovici (1974) showed that a closed set of non-linear, Riccati-type equations can be obtained which describe the depth variations of the impedance elements. In this paper, we complement the study by Abramovici (1974) by deriving the Riccati-type equations for the impedance elements of a 1-D anisotropic medium immediately from the basic field equations and show their relation to the standard matrix propagation formulae. In a separate second part of this study, we discuss a possible extension of the approach based on the Riccati equations to 1-D anisotropic models excited by a generally non-uniform source field.

The structure of the paper is as follows: In Section 2, we present an elementary derivation of the generalized Riccati equations for a 1-D anisotropic medium, and show their relation to the standard matrix propagation formulae for anisotropic layered structures. Numerical aspects of solving the Riccati 1-D anisotropic system are discussed in that section as well. Finally, as an application, in Section 3 the Riccati approach is used to simulate MT responses of structures with a variable regional strike. Results of standard MT de- composition techniques applied to the generated model data are discussed.

\section{Riccati Impedance Equations for a 1-D Aniso- tropic Medium}

\subsection{Elemetary derivation}

We assume a model that consists of a horizontally homogeneous halfspace, $z>0$, with the electrical properties specified by a symmetric and positive definite tensor of the electrical conductivity varying in the $z$ direction only, $\boldsymbol{\sigma}=\boldsymbol{\sigma}(z)$. The halfspace $z<0$ is filled with a perfect insulator (air layer). The MT field is excited by a homogeneous electromagnetic plane wave propagating from sources at $z \rightarrow-\infty$ perpendicularly to the surface of the conductive medium. For this specific model setting, general Maxwell's equations reduce to,

$$
\begin{aligned}
& -E_{y}^{\prime}=i \omega \mu_{0} H_{x}, \\
& -H_{y}^{\prime}=J_{x}=\sigma_{x x} E_{x}+\sigma_{x y} E_{y}+\sigma_{x z} E_{z}, \\
& E_{x}^{\prime}=i \omega \mu_{0} H_{y}, \\
& H_{x}^{\prime}=J_{y}=\sigma_{x y} E_{x}+\sigma_{y y} E_{y}+\sigma_{y z} E_{z}, \\
& H_{z}=0, \quad J_{z}=\sigma_{x z} E_{x}+\sigma_{y z} E_{y}+\sigma_{z z} E_{z}=0,
\end{aligned}
$$

where all the horizontal derivatives have vanished due to the symmetry of the model. In (3), the prime denotes the vertical derivative, $d / d z$, and $E_{v}, H_{v}$, and $J_{v}, v \in\{x, y, z\}$, are components of the electrical intensity, magnetic intensity, and current density, respectively. For brevity, we have left out all the obvious arguments in (3), i.e., $z$ and $\omega$ with the fields and $z$ with the conductivities. Equations (3c) show that no vertical magnetic fields and no vertical currents can exist in a 1-D anisotropic medium excited by a homogeneous plane wave, independently of how much the particular form of the conductivity tensor may prefer the vertical direction.

After eliminating $E_{z}$ from (3a) and (3b) by using the identity $J_{z}=0$, those equations can be written in a simple vector form,

$$
\mathbf{E}^{\prime}=\mathbf{D}^{E H} \mathbf{H}, \quad \mathbf{H}^{\prime}=\mathbf{D}^{H E} \mathbf{E},
$$

with

$$
\begin{aligned}
& \mathbf{E}=\left(\begin{array}{l}
E_{x} \\
E_{y}
\end{array}\right), \quad \mathbf{H}=\left(\begin{array}{l}
H_{x} \\
H_{y}
\end{array}\right), \\
& \mathbf{D}^{E H}=i \omega \mu_{0} \mathbf{P}, \quad \mathbf{D}^{H E}=\mathbf{P} \mathbf{\Sigma}, \quad \mathbf{P}=\left(\begin{array}{cc}
0 & 1 \\
-1 & 0
\end{array}\right) .
\end{aligned}
$$

$\boldsymbol{\Sigma}$ is an effective horizontal conductivity tensor with the elements given by

$$
\begin{aligned}
& \Sigma_{x x}=\sigma_{x x}-\sigma_{z z}^{-1} \sigma_{x z}^{2}, \quad \Sigma_{y y}=\sigma_{y y}-\sigma_{z z}^{-1} \sigma_{y z}^{2}, \\
& \Sigma_{x y}=\Sigma_{y x}=\sigma_{x y}-\sigma_{z z}^{-1} \sigma_{x z} \sigma_{y z} .
\end{aligned}
$$

Let us assume the impedance relation in the 1-D anisotropic model to be of a general form $\mathbf{E}=\mathbf{Z H}$, where $\mathbf{Z}$ represents a $2 \times 2$ impedance tensor. Then, by using this impedance ansatz and (4), we have

$$
\begin{aligned}
\mathbf{E}^{\prime} & =\mathbf{D}^{E H} \mathbf{H}=\mathbf{Z}^{\prime} \mathbf{H}+\mathbf{Z} \mathbf{H}^{\prime}=\mathbf{Z}^{\prime} \mathbf{H}+\mathbf{Z} \mathbf{D}^{H E} \mathbf{E} \\
& =\left(\mathbf{Z}^{\prime}+\mathbf{Z} \mathbf{D}^{H E} \mathbf{Z}\right) \mathbf{H},
\end{aligned}
$$


which gives immediately

$$
\mathbf{Z}^{\prime}+\mathbf{Z D}^{H E} \mathbf{Z}=\mathbf{D}^{E H} .
$$

for an arbitrary non-vanishing magnetic field $\mathbf{H}$. In a similar way we can also derive the equivalent admittance form of the vector Riccati equation,

$$
\mathbf{Y}^{\prime}+\mathbf{Y} \mathbf{D}^{E H} \mathbf{Y}=\mathbf{D}^{H E}
$$

where $\mathbf{H}=\mathbf{Y E}$.

Equation (6) is a generalized Riccati vector equation which controls the depth dependence of the impedance tensor for a 1-D anisotropic medium. It can be easily shown that the equations for the individual components of the impedance tensor are

$$
\begin{aligned}
& Z_{x x}^{\prime}+Z_{A} Z_{x x}+\Sigma_{x y} Z_{B}^{2}=0, \\
& Z_{x y}^{\prime}+Z_{A} Z_{x y}+\Sigma_{y y} Z_{B}^{2}=i \omega \mu_{0}, \\
& Z_{y x}^{\prime}+Z_{A} Z_{y x}-\Sigma_{x x} Z_{B}^{2}=-i \omega \mu_{0}, \\
& Z_{y y}^{\prime}+Z_{A} Z_{y y}-\Sigma_{y x} Z_{B}^{2}=0,
\end{aligned}
$$

where two impedance dependent rotation invariants have been introduced,

$$
\begin{aligned}
& Z_{A}=\operatorname{tr}(\mathbf{P} \boldsymbol{\Sigma} \mathbf{Z})=\Sigma_{x y}\left(Z_{x x}-Z_{y y}\right)-\Sigma_{x x} Z_{x y}+\Sigma_{y y} Z_{y x}, \\
& Z_{B}^{2}=\operatorname{det} \mathbf{Z}=Z_{x x} Z_{y y}-Z_{x y} Z_{y x} .
\end{aligned}
$$

These formulae correspond exactly to equations (86) through (89) by Abramovici (1974).

Hence, the vector Riccati equation (6) represents a coupled system of four first-order non-linear differential equations for the components of the impedance tensor $\mathbf{Z}$. These equations are not entirely independent, however. By summing up the equations for $Z_{x x}$ and for $Z_{y y}$, it can be easily shown that those secondary impedance components are identical except for the sign (see Appendix for the exact proof). It means that (i) generally $Z_{x x}+Z_{y y}=0$ everywhere providing this relation can be proved for at least one $z$ within the model, which is true, e.g., in a homogeneous basement of the model, and (ii) only three first-order differential equations are independent, two for the main impedances and one for the secondary impedance, and have to be considered for propagating the complete impedance tensor through the anisotropic medium.

\subsection{Relation to the matrix propagation formulae}

Similarly as in the isotropic case, we can easily demonstrate that the vector Riccati equations (6), or (7), for the impedances, or admittances, respectively, represent a generalization of the well-known matrix propagation formulae for the field components in a 1-D anisotropic layered medium. As the layered problem with general anisotropy has been dealt with many times by various authors, e.g., by O'Brien and Morrison (1967), Reddy and Rankin (1971), Loewenthal and Landisman (1973), and Dekker and Hastie (1980), we will present only a condensed version of the matrix propagation approach here.

By virtue of (4), the horizontal electric field $\mathbf{E}$ in any homogeneous anisotropic layer is a solution of a secondorder differential equation

$$
\mathbf{E}^{\prime \prime}-\mathbf{D}^{E H} \mathbf{D}^{H E} \mathbf{E}=\mathbf{E}^{\prime \prime}+i \omega \mu_{0} \boldsymbol{\Sigma} \mathbf{E}=0 .
$$

Assuming the solution for the individual components of the vector $\mathbf{E}$ to be of an exponential form, $E_{v}=E_{0 v} \exp (k z)$, $v \in\{x, y\}$, and using this ansatz in (9), we arrive at a system of linear equations for the wave numbers $k$,

$$
\begin{aligned}
& \left(k^{2}+i \omega \mu_{0} \Sigma_{x x}\right) E_{0 x}+i \omega \mu_{0} \Sigma_{x y} E_{0 y}=0, \\
& i \omega \mu_{0} \Sigma_{x y} E_{0 x}+\left(k^{2}+i \omega \mu_{0} \Sigma_{y y}\right) E_{0 y}=0 .
\end{aligned}
$$

For this homogeneous system to have a non-zero solution, its determinant has to be zero. This condition gives four complex wave numbers, $\pm k_{1}$ and $\pm k_{2}$, which correspond to four wave modes that can exist within a particular layer,

$$
k_{1,2}^{2}=-\frac{i \omega \mu_{0}}{2}\left[\Sigma_{x x}+\Sigma_{y y} \pm \sqrt{\left(\Sigma_{x x}-\Sigma_{y y}\right)^{2}+4 \Sigma_{x y}^{2}}\right] .
$$

Moreover, the amplitudes of the individual electric modes, $E_{0 x}$ and $E_{0 y}$, are not independent in the anisotropic medium, but are related, in virtue of (10), via

$$
\frac{E_{0 y}}{E_{0 x}}=-\frac{k^{2}+i \omega \mu_{0} \Sigma_{x x}}{i \omega \mu_{0} \Sigma_{x y}}=-\frac{i \omega \mu_{0} \Sigma_{x y}}{k^{2}+i \omega \mu_{0} \Sigma_{y y}} \stackrel{\text { def }}{=} Q .
$$

In the homogeneous basement, which underlies the stratified section of the conductive halfspace, only the two downgoing wave modes (i.e., those with $\operatorname{Re} k<0$ ) can exist for energetic reasons.

In a particular layer, say $l$-th, within the layer stack, the horizontal field components will now be given by

$$
\mathbf{F}_{l}(z) \stackrel{\operatorname{def}}{=}\left(\begin{array}{l}
\mathbf{E}_{l}(z) \\
\mathbf{H}_{l}(z)
\end{array}\right)=\mathbf{M}_{l} \mathbf{X}_{l}(z) \mathbf{C}_{l}, \quad z \in\left(z_{l-1}, z_{l}\right),
$$

where $z_{l-1}$ and $z_{l}$ are the top and bottom boundary of the layer respectively, and $z_{l}-z_{l-1}=h_{l}$ is its thickness. The matrices involved are defined, by virtue of (4) and (11), as

$$
\begin{aligned}
& \mathbf{M}_{l}=\left(\begin{array}{cccc}
1 & 1 & 1 & 1 \\
Q_{l 1} & Q_{l 1} & Q_{l 2} & Q_{l 2} \\
\gamma k_{l 1} Q_{l 1} & -\gamma k_{l 1} Q_{l 1} & \gamma k_{l 2} Q_{l 2} & -\gamma k_{l 2} Q_{l 2} \\
-\gamma k_{l 1} & \gamma k_{l 1} & -\gamma k_{l 2} & \gamma k_{l 2}
\end{array}\right), \\
& \text { where } \gamma=-\frac{1}{i \omega \mu_{0}},
\end{aligned}
$$

$\mathbf{X}_{l}(z)$ is a diagonal matrix, $\mathbf{X}_{l}(z)=\operatorname{diag}\left\{\exp \left(k_{l 1} z\right)\right.$, $\left.\exp \left(-k_{l 1} z\right), \exp \left(k_{l 2} z\right), \exp \left(-k_{l 2} z\right)\right\}$, and $\mathbf{C}_{l}$ is a column vector with four integration constants that correspond to the four wave modes of the electromagnetic field within the layer. Only two of these integration constants, $C_{N 2}$ and $C_{N 4}$, are non-zero in the homogeneous basement of the model. Here, $N$ is the number of layers including the basement.

The integration constants $\mathbf{C}$ for the individual layers can be found by using the boundary conditions on the layer boundaries, which require the continuity of both the horizontal electric and magnetic field components through the interfaces, i.e. $\mathbf{F}_{l}\left(z_{l}\right)=\mathbf{F}_{l+1}\left(z_{l}\right)$. Consequently, in virtue of (12), the following rule can be given for propagating the vectors $\mathbf{C}$ between successive layers in the stack,

$$
\mathbf{C}_{l}=\mathbf{X}_{l}^{-1}\left(z_{l}\right) \mathbf{M}_{l}^{-1} \mathbf{M}_{l+1} \mathbf{X}_{l+1}\left(z_{l}\right) \mathbf{C}_{l+1} .
$$


Using this rule in (12), and applying it recurrently over layers, we can relate the horizontal fields between any two depth levels within the model. In particular, by using the recurrence down to the top boundary of the homogeneous basement, i.e. to the level $z=z_{N-1}$, we can express the horizontal fields at $z$ within the $l$-th layer by means of only the two non-specified basement coefficients $C_{N 2}$ and $C_{N 4}$,

$$
\begin{aligned}
\mathbf{F}_{l}(z) & =\mathbf{T}_{l}\left(z_{l}-z\right) \prod_{j=l+1}^{N-1} \mathbf{T}_{j}\left(h_{j}\right) \mathbf{M}_{N} \mathbf{X}_{N}\left(z_{N-1}\right) \mathbf{C}_{N} \\
& =\mathbf{T}_{l}\left(z_{l}-z\right) \prod_{j=l+1}^{N-1} \mathbf{T}_{j}\left(h_{j}\right) \mathbf{F}_{N}\left(z_{N-1}\right), \quad z \in\left(z_{l-1}, z_{l}\right),
\end{aligned}
$$

where the transition matrix is

$$
\mathbf{T}_{j}\left(h_{j}\right)=\mathbf{M}_{j} \mathbf{X}_{j}\left(-h_{j}\right) \mathbf{M}_{j}^{-1},
$$

or explicitely, with $c_{1,2}=\cosh k_{1,2} h$ and $s_{1,2}=\sinh k_{1,2} h$ introduced for brevity, and with the index $j$ omitted for that once,

$$
\mathbf{T}(h)=\left(\begin{array}{cc}
\mathbf{T}^{E E} & \mathbf{T}^{E H} \\
\mathbf{T}^{H E} & \mathbf{T}^{H H}
\end{array}\right),
$$

with the $2 \times 2$ matrix blocks given, for $\kappa=Q_{1} / Q_{2}$, by

$$
\begin{aligned}
& \mathbf{T}^{E E}=\frac{1}{1-\kappa}\left(\begin{array}{cc}
c_{1}-\kappa c_{2} & -\frac{1}{Q_{2}}\left(c_{1}-c_{2}\right) \\
Q_{1}\left(c_{1}-c_{2}\right) & -\kappa c_{1}+c_{2}
\end{array}\right), \\
& \mathbf{T}^{E H}=\frac{1}{1-\kappa}\left(\begin{array}{c}
\frac{1}{\gamma Q_{2}}\left(\frac{s_{1}}{k_{1}}-\frac{s_{2}}{k_{2}}\right) \frac{1}{\gamma}\left(\frac{s_{1}}{k_{1}}-\kappa \frac{s_{2}}{k_{2}}\right) \\
\frac{1}{\gamma}\left(\kappa \frac{s_{1}}{k_{1}}-\frac{s_{2}}{k_{2}}\right) \frac{Q_{1}}{\gamma}\left(\frac{s_{1}}{k_{1}}-\frac{s_{2}}{k_{2}}\right)
\end{array}\right), \\
& \mathbf{T}^{H E}=\frac{1}{1-\kappa}\left(\begin{array}{cc}
-\gamma Q_{1}\left(k_{1} s_{1}-k_{2} s_{2}\right) & \gamma\left(\kappa k_{1} s_{1}-k_{2} s_{2}\right) \\
\gamma\left(k_{1} s_{1}-\kappa k_{2} s_{2}\right) & -\frac{\gamma}{Q_{2}}\left(k_{1} s_{1}-k_{2} s_{2}\right)
\end{array}\right), \\
& \mathbf{T}^{H H}=\frac{1}{1-\kappa}\left(\begin{array}{cc}
-\kappa c_{1}+c_{2} & -Q_{1}\left(c_{1}-c_{2}\right) \\
\frac{1}{Q_{2}}\left(c_{1}-c_{2}\right) & c_{1}-\kappa c_{2}
\end{array}\right) .
\end{aligned}
$$

The obvious meaning of the transition matrix $\mathbf{T}_{j}\left(h_{j}\right)$ is that it relates the fields on the top and on the bottom of the $j$-th layer, $\mathbf{F}_{j}\left(z_{j-1}\right)=\mathbf{T}_{j}\left(h_{j}\right) \mathbf{F}_{j}\left(z_{j}\right)$. The latter expression allows us to find a similar relation for the impedance tensors as well. With a simplified notation $\mathbf{Z}\left(z_{j}\right) \equiv \mathbf{Z}_{j}$, we can easily show

$$
\begin{aligned}
\mathbf{E}_{j}\left(z_{j-1}\right) & =\mathbf{Z}_{j-1} \mathbf{H}_{j}\left(z_{j-1}\right) \\
& =\mathbf{Z}_{j-1}\left[\mathbf{T}_{j}^{H E}\left(h_{j}\right) \mathbf{Z}_{j}+\mathbf{T}_{j}^{H H}\left(h_{j}\right)\right] \mathbf{H}_{j}\left(z_{j}\right) \\
& =\left[\mathbf{T}_{j}^{E E}\left(h_{j}\right) \mathbf{Z}_{j}+\mathbf{T}_{j}^{E H}\left(h_{j}\right)\right] \mathbf{H}_{j}\left(z_{j}\right),
\end{aligned}
$$

which provides the propagation formula for the impedance tensor in the form

$$
\begin{aligned}
\mathbf{Z}_{j-1}= & {\left[\mathbf{T}_{j}^{E E}\left(h_{j}\right) \mathbf{Z}_{j}+\mathbf{T}_{j}^{E H}\left(h_{j}\right)\right] } \\
& \cdot\left[\mathbf{T}_{j}^{H E}\left(h_{j}\right) \mathbf{Z}_{j}+\mathbf{T}_{j}^{H H}\left(h_{j}\right)\right]^{-1} .
\end{aligned}
$$

This formula is a generalization of (2) to anisotropic layers, and can be easily shown to degenerate into (2) if the conductivity reduces to isotropic.

To generalize (15) to continuously varying anisotropic conductivities, we can proceed similarly as in the isotropic case, and analyze (15) for $h_{j} \equiv \Delta z \rightarrow 0$. In this case, $s=\sinh k \Delta z \approx k \Delta z$ and $c=\cosh k \Delta z \approx 1$, if terms of the order higher than one in $\Delta z$ are neglected. Then, both $\mathbf{T}^{E E}$ and $\mathbf{T}^{H H}$ reduce to $2 \times 2$ unit matrices, and the anti-diagonal blocks of $\mathbf{T}$ are approximately

$$
\begin{aligned}
\mathbf{T}^{E H} & \approx \gamma^{-1} \Delta z \mathbf{P}, \\
\mathbf{T}^{H E} & \approx \frac{\gamma \Delta z}{1-\kappa}\left(\begin{array}{cc}
-Q_{1}\left(k_{1}^{2}-k_{2}^{2}\right) & \kappa k_{1}^{2}-k_{2}^{2} \\
k_{1}^{2}-\kappa k_{2}^{2} & -Q_{2}^{-1}\left(k_{1}^{2}-k_{2}^{2}\right)
\end{array}\right) \\
& =-\Delta z \mathbf{P} \boldsymbol{\Sigma},
\end{aligned}
$$

the last simplification being a consequence of our having applied the following elementary identities

$$
\begin{aligned}
& Q_{1} Q_{2}=-1, \\
& k^{2}=\gamma^{-1}\left(\Sigma_{x x}+Q \Sigma_{x y}\right)=\gamma^{-1}\left(\Sigma_{y y}+Q^{-1} \Sigma_{x y}\right), \\
& k_{1}^{2}-k_{2}^{2}=-\gamma^{-1}(1-\kappa) Q_{2} \Sigma_{x y},
\end{aligned}
$$

which all follow immediately from (11).

Replacing now in (15) $\mathbf{Z}_{j}$ and $\mathbf{Z}_{j-1}$ by $\mathbf{Z}(z+\Delta z)$ and $\mathbf{Z}(z)$, respectively, and using the above approximations, we can modify (15) into

$$
\begin{gathered}
\frac{\mathbf{Z}(z+\Delta z)-\mathbf{Z}(z)}{\Delta z}+\mathbf{Z}(z) \mathbf{P} \boldsymbol{\Sigma} \mathbf{Z}(z+\Delta z) \\
=-\gamma^{-1} \mathbf{P},
\end{gathered}
$$

which gives exactly the vector Riccati equation (6) if $\Delta z \rightarrow$ 0 .

\subsection{Numerical solution}

The Riccati vector equation (6) represents a system of four, or three independent, coupled first-order ordinary differential equations for the impedance elements of a 1-D anisotropic medium. Any standard ODE solver can be applied to solve this system numerically. In this study, we have used two techniques to solve (6), specifically

(i) a simple implicit stepping from the basement through the medium, analogous to that often used for the isotropic problem, based on the difference approximation of the spatial derivative of $\mathbf{Z}$ according to the formula (16),

$$
\begin{aligned}
\mathbf{Z}(z)= & {\left[\mathbf{Z}(z+\Delta z)-\mathbf{D}^{E H} \Delta z\right] } \\
& \cdot\left[\mathbf{1}-\mathbf{D}^{H E} \mathbf{Z}(z+\Delta z) \Delta z\right]^{-1},
\end{aligned}
$$

where 1 is a $2 \times 2$ unit matrix. We assume that the model is underlain by a homogeneous, but generally anisotropic halfspace, which provides us with the starting impedance at the top, or anywhere within the basement, given by

$$
\begin{aligned}
\mathbf{Z}_{\text {base }}= & -\frac{i \omega \mu_{0}}{1-\kappa} \\
& \cdot\left(\begin{array}{cc}
-Q_{1}\left(k_{1}^{-1}-k_{2}^{-1}\right) & k_{1}^{-1}-\kappa k_{2}^{-1} \\
\kappa k_{1}^{-1}-k_{2}^{-1} & Q_{1}\left(k_{1}^{-1}-k_{2}^{-1}\right)
\end{array}\right),
\end{aligned}
$$


with $k_{1}, k_{2}, Q_{1}$, and $\kappa$ corresponding to the electrical parameters of the basement.

Physically, this numerical procedure is equivalent to the matrix propagation method applied to a medium obtained by approximating the 1-D structure by many thin homogeneous layers. If no adaptive step is attempted in the integration, the procedure can sometimes lead to excessive computations. Especially in case of high frequencies for models with only few zones of rapid conductivity changes (e.g., jumps through layer interfaces), the integration step must be chosen small enough to properly resolve rapid impedance variations around the boundaries, and a plethora of computation steps is then spent to move across homogeneous, or only slowly varying portions of the conductivity section.

(ii) Alternatively, we used a more advanced Livermore ODE solver LSODA from the public Netlib repository. This routine is implemented as a double precision Fortran numerical ODE solving procedure with adaptive step size and accuracy control, and an automatic switching for stiff and non-stiff ODE systems (Hindmarsh, 1983; Petzold, 1983). As (6) does not involve solution modes with largely different rates of the depth variation, the Riccati system is non-stiff, and the above procedure defaults to the Adams predictorcorrector method (Ralston, 1965).

In Fig. 1, we present an example of using the latter numerical procedure to the solution of (6) for a simple layered

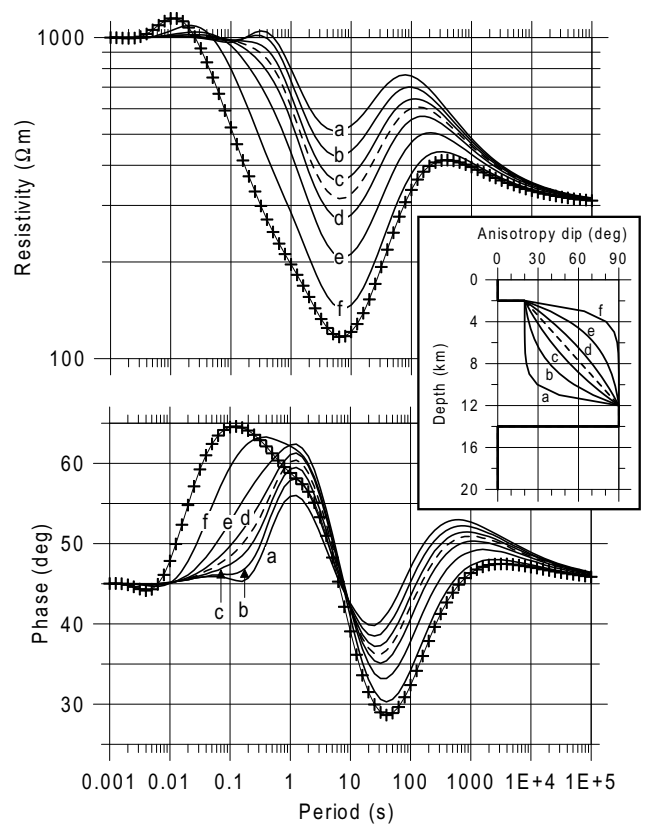

Fig. 1. Resistivity and phase $y x$-curves for an anisotropic layered model with exponentially increasing anisotropy dip within an upper crustal layer. See text for the particular model parameters. The rate of change of the anisotropy dip for different models is shown in the inset. The dashed line corresponds to the linear increase of the dip with depth. The crosses show the $x y$-curves in the direction parallel to the strike. Notice the scale exaggeration on the vertical axes. model with one anisotropic layer. The layer thicknesses in the 5-layer model are 2,10, 2, 100, and $\infty \mathrm{km}$. The resistivities of the isotropic layers are $\varrho_{1}=1000 \Omega \mathrm{m}, \varrho_{3}=$ $100 \Omega \mathrm{m}, \varrho_{4}=1000 \Omega \mathrm{m}$, and $\varrho_{5}=300 \Omega \mathrm{m}$. The second layer is anisotropic with the principal resistivities $\varrho_{2, \min }=$ $100 \Omega \mathrm{m}, \varrho_{2, \max }=1000 \Omega \mathrm{m}$ in the $(y, z)$-coordinate plane, and with a smoothly varying anisotropy dip defined here as a deviation of the minimum resistivity direction from the vertical axis $z$. The anisotropy dip varies exponentially from $20^{\circ}$ at the top of the layer to $90^{\circ}$ at its bottom, with different rates of change for different models $a$ through $f$ (Fig. 1). The resistivity along the $x$-axis is equal to $\varrho_{2 \text {,min }}$. The medium may be considered a schematic model of a lystric structure with curved strike-parallel conductive planes within the anisotropic layer.

For the above 1-D anisotropic model, the variations in the anisotropy dip are principally indistinguishable from appropriately scaled vertical conductivity variations. For the anisotropy dip $\alpha_{D 2}$ (measured from the vertical axis here), the conductivity tensor in the anisotropic layer 2 is given by

$$
\begin{aligned}
\boldsymbol{\sigma}_{2}= & \mathbf{R}_{x}\left(\alpha_{D 2}-90^{\circ}\right) \operatorname{diag}\left\{\varrho_{2, \min }^{-1}, \varrho_{2, \min }^{-1}, \varrho_{2, \max }^{-1}\right\} \\
& \cdot \mathbf{R}_{x}\left(90^{\circ}-\alpha_{D 2}\right),
\end{aligned}
$$

where $\mathbf{R}_{x}(\alpha)$ is the elementary rotation matrix around the $x$-axis by $\alpha$. From this formula, the components of the effective horizontal conductivity tensor $\Sigma_{2}(5)$ can be computed directly,

$$
\begin{aligned}
& \Sigma_{2, x x}=\varrho_{2, \min }^{-1}, \\
& \Sigma_{2, y y}=\left(\varrho_{2, \max } \cos ^{2} \alpha_{D 2}+\varrho_{2, \min } \sin ^{2} \alpha_{D 2}\right)^{-1}, \\
& \Sigma_{2, x y}=0 .
\end{aligned}
$$

Thus, the equivalent horizontal resistivity in the $y$-direction is $\varrho_{y 2}=\varrho_{2, \max } \cos ^{2} \alpha_{D 2}+\varrho_{2, \min } \sin ^{2} \alpha_{D 2}$.

The curves in Fig. 1 conform with the above equivalency principle. With the decreasing rate of the exponential variation of the anisotropy dip (i.e., from $a$ towards $f$ in Fig. 1) the portion of the anisotropic layer with smaller resistivity $\varrho_{y 2}$ becomes larger, starting from the bottom of the layer. Consequently, the $y x$-curves gradually approach the limit case of the strike-parallel $x y$-curve (crosses in Fig. 1) that corresponds to the constant resistivity $\varrho_{x 2}=\varrho_{2, \text { min }}$ throughout the layer.

\section{Application: Synthetic Model Study on the MT Decomposition for a Variable Strike Direction 3.1 Separated homogeneous layers with different strikes}

Anisotropic layered models can be used to simulate structures with directional characteristics variable with depth. In magnetotellurics, the applicability of a 3-D local/2-D regional composite model of the medium is verified by evaluating a set of decomposition parameters (Groom and Bailey, 1989; Bahr, 1991) and by checking on their frequency independence within a certain period range. In practice, situations are not rarely encountered when either different sets of decomposition parameters can be identified within different period bands, or when the parameters display systematic variations with the period. Those situations 

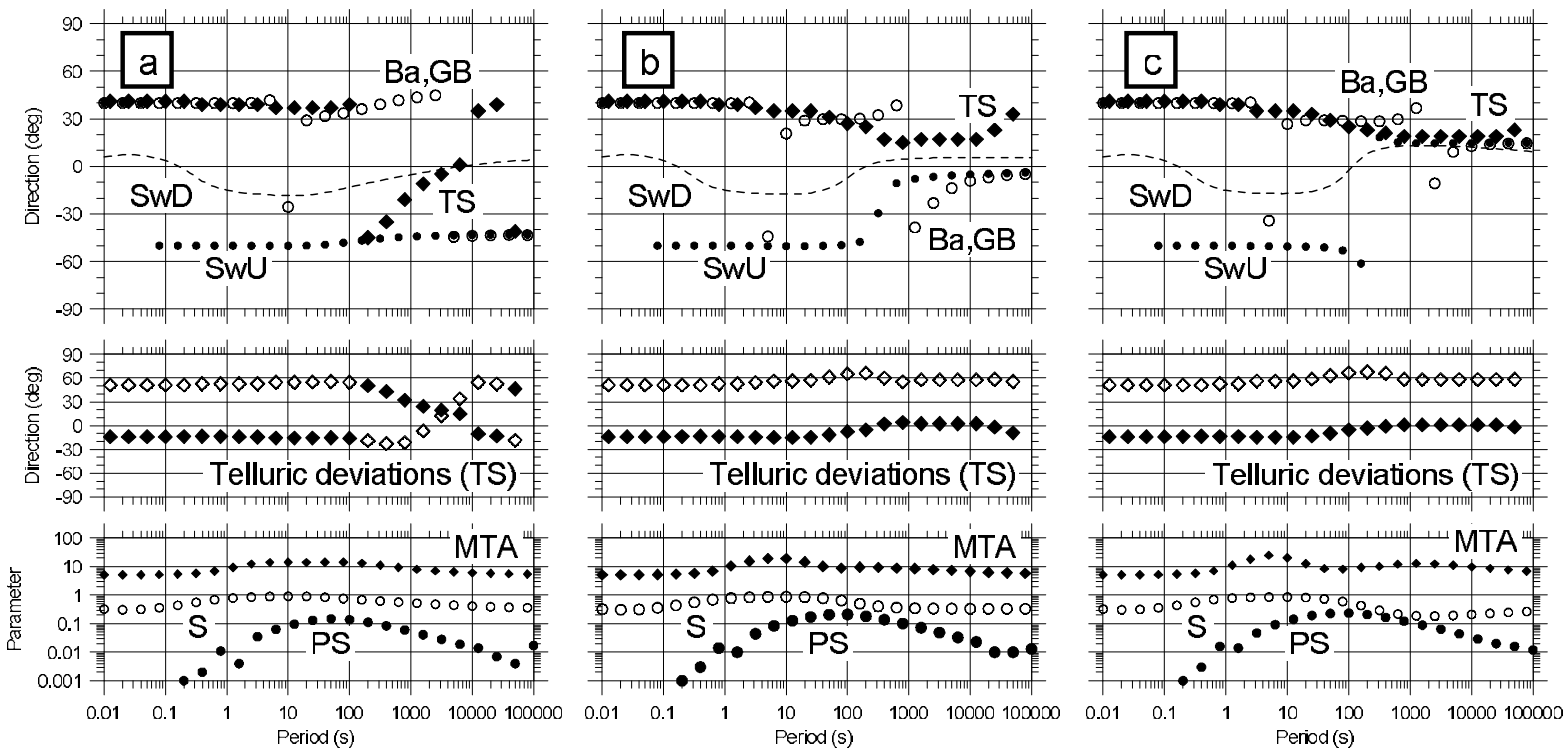

Fig. 2. Results of the MT decomposition analysis applied to a distorted 5-layer model specified in the text. The panels $a, b, c$ correspond to different minimum resistivities of the deep anisotropic layer. The top panels show the characteristic directions for the individual cases: SwD - Swift's direction for the distorted impedances, SwU-Swift's direction for the corresponding undistorted data, Ba, GB — regional strike recovered from the distorted data by Bahr's and Groom and Bailey's decomposition procedures, TS - regional strike obtained from the distorted data by Smith's decomposition procedure with magnetic distortions considered. The middle panels show the telluric deviations computed for the direction predicted by Smith's decomposition procedure. According to Bahr (1991), the telluric deviations are defined by $\tan \beta_{x}=Z_{y y} / Z_{x y}$ and $\tan \beta_{y}=-Z_{x x} / Z_{y x}$ with impedances considered in the direction of the regional strike. They are related to inverse tangents of Groom and Bailey's (1989) (angular) twist and shear parameters by $\beta_{x}=\beta_{T W}+\beta_{S H}$ and $\beta_{y}=\beta_{T W}-\beta_{S H}$. The bottom panels show relevant MT parameters: MTA-MT impedance anisotropy, S-skew, PS-Bahr's phase sensitive skew.

are often interpreted in terms of a depth-variable regional strike, although particularly the latter case may just indicate that a fully 3-D regional structure is involved, and no regional strike exists at all. In this respect, numerical simulations may be a valuable source of information for assessing the manifestion of depth-variable directional properties of the electrical conductivity in the surface MT data.

Though powerful 3-D modelling codes are available today, it is still rather difficult to simulate media with oblique 2-D structures located at different depths within the section. In this respect, anisotropic layered media represent extremely simple models in which the variable strike direction can be simulated by variations of the preferred conductivity direction through the layers. It must be noticed, however, that the analogy between genuinely heterogeneous structures and anisotropic layered media is not complete. Two aspects have to be remembered that restrict the complete parallelism between those cases. First, while a shallow 2-D structure becomes a part of the local distorting structure for long enough periods, which results in a permanent change of the distortion matrix, the effect of a shallow anisotropic layer gradually disappears from the surface data as the induction space becomes larger. Due to the symmetry of the 1-D problem, any surface and/or volume charges that can arise in the layer due to its general anisotropy do not show any lateral gradients and, consequently, cannot contribute to static distortions of the telluric field. Second, an undistorted 1-D anisotropic medium, however complex its conductivity variation may be, always produces MT data with the skew equal to zero. Consequently, characteristic 3-D induction phenomena at the transition between two oblique heteroge- neous structures cannot be properly simulated by using a 1-D anisotropic model.

Our first synthetic study deals with a model involving two separated homogeneous anisotropic layers with different anisotropy strikes, which simulate two different structural strikes at different depths. The model data are further distorted by an arbitrary constant static shift matrix A throughout the period range considered. The layer thicknesses in the 5-layer model considered are, from the top down to the basement, $3,2,10,30$, and $\infty \mathrm{km}$. The corresponding resistivities are $\varrho_{1}=1000 \Omega \mathrm{m}, \varrho_{3}=1000 \Omega \mathrm{m}, \varrho_{5}=500 \Omega \mathrm{m}$; the second and fourth layers are anisotropic with the resistivity parameters $\varrho_{2, \min }=3 \Omega \mathrm{m}, \varrho_{2, \max }=300 \Omega \mathrm{m}$, $\alpha_{S 2, \min }=40^{\circ}$, and $\varrho_{4, \max }=300 \Omega \mathrm{m}, \alpha_{S 4, \min }=-70^{\circ}$, where the anisotropy strike $\alpha_{S \text {, min }}$ indicates the direction of the minimum resistivity in the horizontal direction with respect to the $(x, z)$-coordinate plane. The minimum resistivity of the fourth layer is chosen from $\varrho_{4, \min }=100 \Omega \mathrm{m}$ (a), $30 \Omega \mathrm{m}$ (b), $10 \Omega \mathrm{m}$ (c), to analyze the effect of different conductance ratios of the shallow and deep anisotropic structures involved. The elements of the static distortion ma$\operatorname{trix} \mathbf{A}$ are $a_{x x}=2.5, a_{x y}=-0.3, a_{y x}=0.7, a_{y y}=0.5$. This telluric distortion is characterized by an elongated distortion ellipse with the major axis at $14.9^{\circ}$ with respect to $x$ and with the major and minor semi-axes of 2.60 and 0.56 respectively. For simplicity, we have not contaminated the model data with any additional noise here.

In Fig. 2, we present results of the MT decomposition analysis applied to the data from the above models. The individual plots show Swift's directions, both for the original, undistorted and distorted models, regional strikes predicted 
(a)

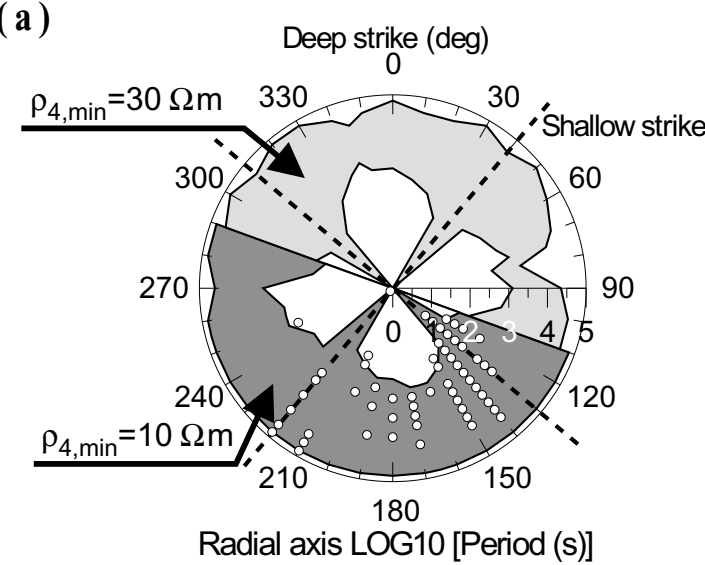

(b)

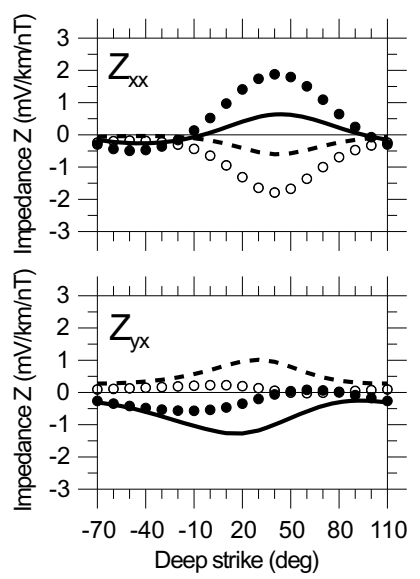

Fig. 3. (a) Frequency resolution of Smith's (1997) decomposition procedure with respect to the regional strike of the deep anisotropic layer. The anisotropy strike of the shallow layer is $\alpha_{S 2}=40^{\circ}$. See the text for the other model parameters. For the deep anisotropy strikes from the interval of $-70^{\circ}$ to $110^{\circ}$, the gray zones show period ranges for which the true deep strike has been retrieved from noise-free data within $5^{\circ}$. The light gray zone correponds to the model with $\varrho_{4, \min }=30 \Omega \mathrm{m}$, the dark gray zone is a corresponding mirror image for the model with $\varrho_{4, \text { min }}=10 \Omega \mathrm{m}$. For the latter model, the white circles show point-wise the same results obtained from noisy impedances ( $2 \%$ of normal noise added to the model data). (b) Variations of the undistorted (full lines - Re $Z$, dashed lines - $\operatorname{Im} Z$ ) and distorted (full circles - $\operatorname{Re} Z_{\text {dist }}$, empty circles - Im $Z_{\text {dist }}$ ) impedance elements for the latter model as a function of the deep anisotropy strike $\alpha_{S 4}$ for the period of $500 \mathrm{~s}$.

by Bahr's (1991) and Groom and Bailey's (1989) decomposition procedures, as well as regional strikes provided by Smith's (1997) procedure, which considers also magnetic distortions in the data. Further, telluric deviations (Bahr, 1991) are shown for the strike direction obtained from the last-named decomposition technique, and basic MT parameters, specifically the impedance anisotropy, skew, and Bahr's phase sensitive skew, are displayed as well.

Common features of all three models are: (i) Swift's direction computed from the distorted data $(\mathrm{SwD})$ is largely affected by the distortions, and does not allow us to draw any immediate conclusion as to the strike variations within the model, except for their likely presence. Only for the model (c), with a very low minimum resistivity of the deep anisotropic layer, the distorted Swift's direction is clearly attracted by that layer at long periods. The influence of the distortion is similarly expressed by the classical skew parameter (S), which is generally greater than 0.3 all over the period range considered. (ii) The shallow anisotropic layer is equally well resolved by all decomposition procedures (regional strike of $40^{\circ}$ for periods up to several seconds). Within the relevant period range, the decomposition parameters are stable and correspond to those resulting directly from the distortion matrix (telluric deviations of $52.1^{\circ}$ and $-13.5^{\circ}$ for the regional strike of $40^{\circ}$ ). The phase sensitive skew (PS) is less than 0.05 over the whole relevant period range, indicating that the composite model with a 2-D regional structure is a good approximation to the real situation. (iii) For the longest periods considered, typically greater than several thousands of seconds, the classical decomposition routines give a regional strike that approaches Swift's direction for the undistorted model (SwU). It means that they satisfactorily remove the distortion effect from the data and provide the same directional information as the data without distortions, but they fail to retrieve the true regional strike at depth.
Interesting behaviour displays the regional strike derived from Smith's (1997) decomposition procedure (TS). For models in Fig. 2(b), (c), this regional strike estimate approaches the correct regional strike within the period range of several hundreds of seconds to more than $10000 \mathrm{~s}$, which can be, by variations in all the parameters considered, identified with the range of manifestation of the deep anisotropic layer. In many runs with various double-strike models we could verify this observation for a broad range of model parameters provided the ratio of the maximum conductances of the two anisotropic layers is large enough for the deep layer to be sensed reliably. It is, however, not clear whether this behaviour of the TS-regional strike is specific of anisotropic 1-D structures only, or whether it can be expected to apply to laterally heterogeneous media with a variable strike as well.

Owing to the electrical anisotropy in the shallow part of the section, an induced anisotropy of the resolution of the surface data to deeper structures can be expected as well. To assess this effect, we repeated the decomposition analysis for the above model with a varying anisotropy strike of the deep layer within the range $\alpha_{S 4} \in\left(-70^{\circ}, 110^{\circ}\right)$. For accurate data, the variable strike $\alpha_{S 4}$ affects the width of the period range in which the TS-strike provides a satisfactory estimate of the true deep strike. In Fig. 3(a), we show that range for two versions of the model, one with $\varrho_{4, \min }=30 \Omega \mathrm{m}$ (light gray diagram) and the other with $\varrho_{4, \min }=10 \Omega \mathrm{m}$ (dark gray diagram, mirror image). The grayed zones indicate periods for which the TS-strike equals to the true deep strike within $5^{\circ}$ and the telluric deviations do not show noticable frequency variations. Obviously, for $\alpha_{S 4}=-50^{\circ}$ and $\alpha_{S 4}=40^{\circ}$ the strikes of the shallow and deep layer are aligned (with $90^{\circ}$ ambiguity considered), and one single strike is recovered practically all over the data range. For oblique anisotropy strikes, the recovery range is narrowing, especially for the smaller contrast between the maximum 
conductances of the two anisotropic layers. The long period boundary of the recovery range is somehow diffuse as the composite model fits the long period impedances with high accuracy for a broad range of the regional strike values.

The rate of success in estimating the deep strike drops dramatically for noisy data. The white points in Fig. 3(a) show periods for which the regional strike has been retrieved within $5^{\circ}$ of its true value from the model data after $2 \%$ of normal noise was added to the impedance elements. For the model with the less conductive deep layer, practically no reliable estimates have been obtained in the noisy case. Even in the case of the more conductive deep layer, with the maximum conductance ratio of the deep and shallow aniso-

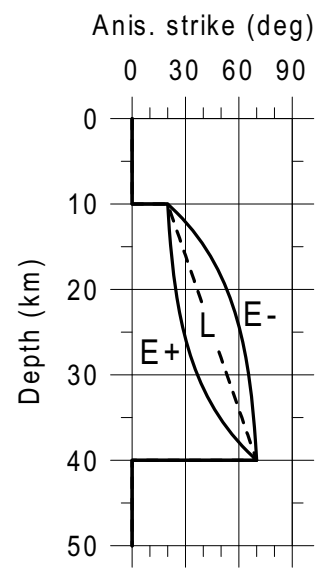

Fig. 4. Exponentially varying anisotropy strike within the second layer of a 3-layer model described in the text. The depth variations of the strike are described by $\alpha_{S 2}(z)=A \exp (f z)+B$, or by a linear function of $z$. For the cases $\mathrm{E}$ - and $\mathrm{E}+$, the exponential factors are, in $\mathrm{m}^{-1}, f=-0.0003$, and $f=0.0003$, respectively. $\mathrm{L}$ is for a linear depth-dependence of $\alpha_{S 2}(z)$. tropic layer of $S_{2, \max } / S_{4, \max }=4.5$, the deep regional strike could not be detected at all for $\alpha_{S 4}$ from within the quadrant $\left(20^{\circ}, 110^{\circ}\right)$. The explanation is that the specific distortion matrix applied to the model data causes one of the main impedance elements, specifically $Z_{y x}$, to almost vanish for those anisotropy strikes (Fig. 3(b)). Hence, any procedure involving implicitely a rotation of the noisy impedance tensor necessarily provides highly scattered and inconsistent results over the respective range of the anisotropy strikes.

\subsection{Smoothly varying regional strike}

The manifestation of a smoothly varying regional strike in MT data, and in particular in the decomposition parameters, is another problem that can be simulated by using 1-D anisotropic models. Of course, the above mentioned limitations of anisotropic models as compared with true laterally inhomogeneous structures must be remembered in this case, too.

For a depth-variable strike analysis, we studied a simple model consisting of three layers with thicknesses 10, 30, and $\infty \mathrm{km}$. The resistivities of the isotropic first and third layer were $\varrho_{1}=1000 \Omega \mathrm{m}, \varrho_{3}=500 \Omega \mathrm{m}$, and the principal resistivities of the azimuthally anisotropic second layer were $\varrho_{2, \min }=\varrho_{2 z}=30 \Omega \mathrm{m}$ and $\varrho_{2, \max }=300 \Omega \mathrm{m}$. The anisotropy strike in the second layer varies smoothly from $20^{\circ}$ at the top of the layer to $70^{\circ}$ at its bottom according to an exponential law (Fig. 4). Aditionally, we multiplied the model impedance matrix by the real and constant distortion matrix which was already used in the previous section. Further, only noise-free data are considered.

In Fig. 5, we show the decomposition parameters for the three variants of the model from Fig. 4. Independently of the particular shape of the depth dependence of the anisotropy strike, all regional strike estimates approach the undistorted Swift's direction, both for short and long period ranges. For short periods, the strike at the top of the transition layer is
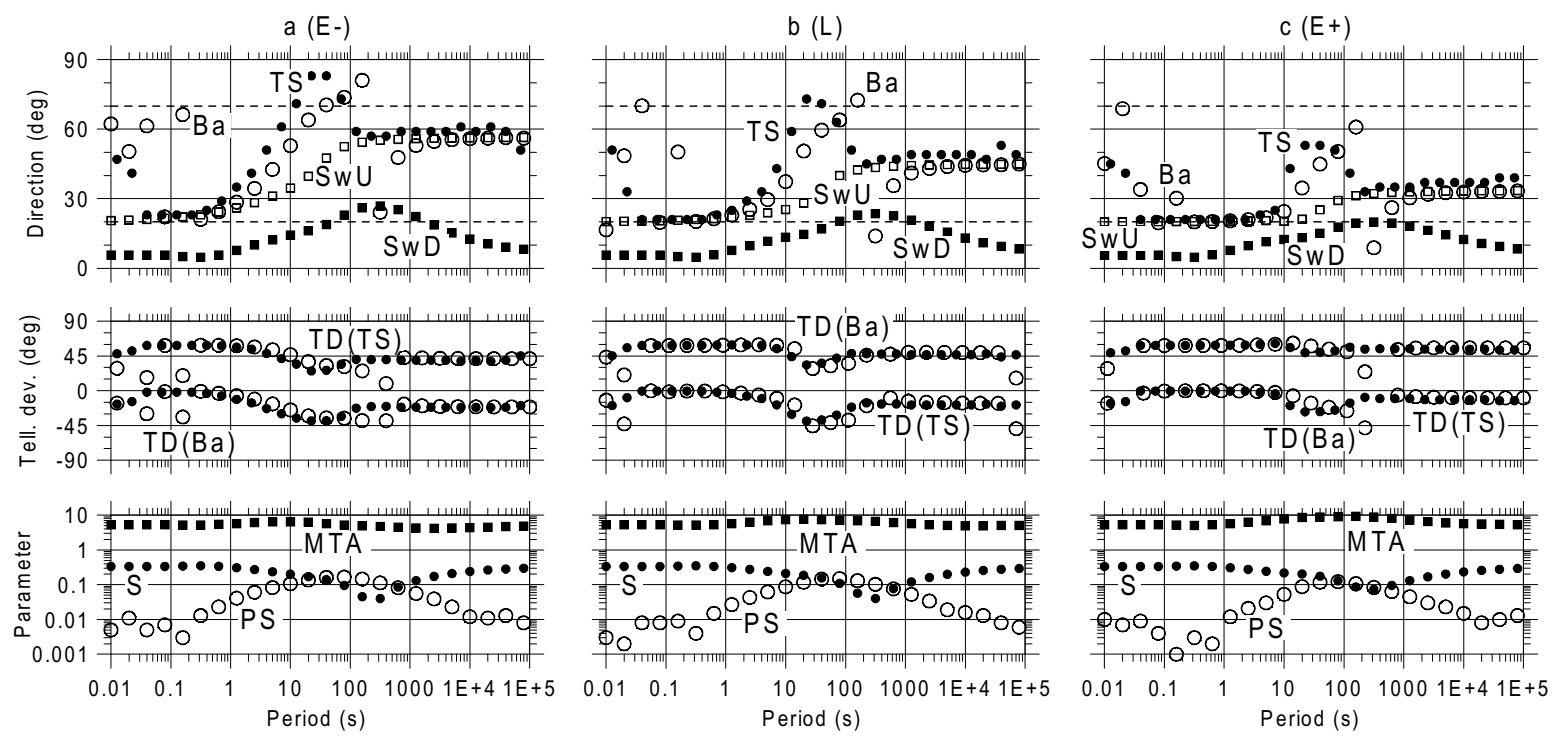

Fig. 5. Decomposition parameters for noise-free data generated by models with a smoothly varying anisotropy strike. See the text for the model parameters. The respective anisotropy strike functions are shown in Fig. 4. For the symbols used, see Fig. 3 caption. Additional symbols: TD(Ba) - telluric deviations for Bahr's regional strike estimate (empty circles in the middle panels), TD(TS) - telluric deviations for the strike estimate according to Smith (full circles in the middle panels). 
retrieved correctly. The long period Swift's direction from undistorted data depends on how fast the anisotropy strike approaches its bottom limit. For variations with negative exponents, the anisotropy strike is close to its bottom value over a thicker portion of the layer and, consequently, both the undistorted Swift's direction and regional strike estimates are closer to this value as well. Contrary, for the positive exponent of $f=0.0003$ in Fig. $5 \mathrm{c}$, the discrepancy between the bottom anisotropy strike and Swift's undistorted direction is almost $40^{\circ}$ at long periods.

The transition zone itself is sensed by the data within the period range of about 10 to $1000 \mathrm{~s}$. The decomposition parameters are frequency dependent in this range. The singlefrequency regional strike estimates do not follow, however, a simple transition law. They rather display an instability, characterized by fast changes, or jumps, of the respective estimates over the whole transition range.

The noise in the data largely obscures the results of the decomposition simulations for models with a depth-variable strike. It is hardly suprising in view of all the experience accumulated from a lot of experiments that tried to detect just a single regional strike in practical MT data (e.g., Jones and Groom, 1993).

\section{Conclusion}

The Riccati equation approach to evaluating the MT impedances, or admittances, in a 1-D horizontally homogeneous medium has been for long an alternative to matrix or impedance propagation formulae, derived for layered structures. Though an analytical solution to the Riccati nonlinear equation exists for only few specific cases of the conductivity profiles, its numerical solution is extremely simple if a starting impedance is known, e.g., in the homogeneous basement of the model. The Riccati equation approach allows us to naturally consider both the discrete layered media and models with a piecewise smoothly varying conductivity distribution.

For 1-D anisotropic media, the idea of using first-order differential equations to compute the MT impedances goes back to Abramovici (1974). We have demonstrated here that analogues of the Riccati equation for the anisotropic case can be obtained readily from the basic field equations. Similarly as in the isotropic case, these equations represent a limiting case of the impedance propagation formulae for anisotropic layered media if layer thicknesses approach zero. The Riccati equations in the 1-D anisotropic case form a system of four coupled non-linear first-order differential equations for the impedance elements (6). Only three of those equations are independent due to the internal symmetry condition $Z_{x x}+Z_{y y}=0$. The Riccati system can be easily solved numerically by applying practically any standard ODE solver. By using the Riccati approach, the modelling of 1-D structures can start immediately by simple numerical tools, without going through the complex algebra involved in the matrix and impedance propagation procedures.

Depth variable conductivity and anisotropy conditions can be used to partly simulate transitions between earth structures with different strikes at different depths. Though the analogy between the 1-D anisotropic media and laterally heterogeneous structures with a depth-variable strike is not complete, especially as regards the changes of the distortion parameters with decreasing frequency, simple simulations with anisotropic stratified models may show whether strike variations with depth are principally detectable in surface data. Similar synthetic experiments were made earlier, e.g., by Smith (1997) who tested his decomposition scheme with magnetic distortions considered on a model with two anisotropic layers, one shallow for near-surface effects, and the other deep to simulate the regional strike. Santos and Mendes-Victor (2000) compared in detail Groom and Bailey's decomposition parameters for anisotropic models with static distortions added artificially. While for models with one single anisotropic layer the impedance tensor properties are similar to those produced by distorted 2-D structures, a joint effect of a shallow and deep anisotropy results in a variety of situations that depend largely on the depth, thickness, and conductivity anisotropy of the shallow layer. Our experiments show that in a multi-layer anisotropic case and for noise-free data the traditional regional strike estimates by Groom and Bailey (1989) and Bahr (1991) approach Swift's direction of the undistorted model in the long period range, i.e., they provide the same directionality information which could be inferred from the data without static distortions. For well separated anisotropic layers, the decomposition procedure that considers the static distortions of the magnetic components as well (Smith, 1997) seems to better retrieve the true regional strikes of the individual layers provided the deeper layers can be resolved sufficiently well within the section. As yet it has not been clarified whether this behaviour is typical of anisotropic stratified media only, or whether laterally inhomogeneous structures with a depth-variable strike show similar features.

Strike transitions between various depth levels within a 1-D anisotropic section are often expressed by more irregular variations of the decomposition parameters. In particular, the regional strike estimates may show excessive changes and jumps within period bands that correspond to deep strike changes.

For noise-free data, the regional directionality conclusions are largely independent of the particular distortion parameters. With even a small amount of noise in the data, however, the resolution with respect to the deep strike variations gets largely reduced. Moreover, the particular distortion parameters involved highly influence the way in which the data errors propagate in rotated impedances, so that substantial dependence of the directionality conclusions on the particular distortion parameters appears.

Acknowledgments. The financial support provided by the Grant Agency of the Czech Republic, through the grants Nos. 205/99/0917 and 205/99/0907, is gratefully acknowledged. Additional support was also furnished by the Ministry of Education, Youth and Sports of the Czech Republic under the contract ME185 within the Czech-Japanese Cooperation in R\&D initiative. Moreover, we thank reviewers Mark Everett and Peter Weidelt for their helpful and valuable comments and suggestions for improving the original manuscript.

\section{Appendix A. $Z_{x x}+Z_{y y}=0$ in the Anisotropic Lay- ered Medium}

From (8), the sum of the secondary impedances, $S_{1} \equiv$ 
$Z_{x x}+Z_{y y}$, is controlled by the differential equation

$$
S_{1}^{\prime}+Z_{A} S_{1}=0 \text {. }
$$

To evaluate the invariant $Z_{A}$ in (A.1) explicitely, we express the impedance tensor in a 1-D anisotropic medium by means of the horizontal field components obtained for two independent field polarizations,

$\mathbf{Z}=\frac{1}{D_{H}}\left(\begin{array}{cc}E_{x}^{(1)} H_{y}^{(2)}-H_{y}^{(1)} E_{x}^{(2)} & H_{x}^{(1)} E_{x}^{(2)}-E_{x}^{(1)} H_{x}^{(2)} \\ E_{y}^{(1)} H_{y}^{(2)}-H_{y}^{(1)} E_{y}^{(2)} & H_{x}^{(1)} E_{y}^{(2)}-E_{y}^{(1)} H_{x}^{(2)}\end{array}\right)$,

with $D_{H}=H_{x}^{(1)} H_{y}^{(2)}-H_{y}^{(1)} H_{x}^{(2)}$ and the superscripts (1) and (2) specifying the individual polarizations. Now, by using the impedance elements from (A.2) in the definition formula for $Z_{A}$ we easily obtain

$$
\begin{aligned}
Z_{A} & =\Sigma_{x y}\left(Z_{x x}-Z_{y y}\right)-\Sigma_{x x} Z_{x y}+\Sigma_{y y} Z_{y x} \\
& =D_{H}^{-1}\left(\mathbf{J}^{(1)} \cdot \mathbf{H}^{(2)}-\mathbf{J}^{(2)} \cdot \mathbf{H}^{(1)}\right) \\
& =D_{H}^{-1}\left(\mathbf{H}^{(2)} \cdot \nabla \times \mathbf{H}^{(1)}-\mathbf{H}^{(1)} \cdot \nabla \times \mathbf{H}^{(2)}\right) \\
& =D_{H}^{-1} \nabla \cdot\left(\mathbf{H}^{(1)} \times \mathbf{H}^{(2)}\right),
\end{aligned}
$$

where Maxwell's equation $\nabla \times \mathbf{H}=\mathbf{J}$ and the vector identity $\nabla \cdot(\mathbf{a} \times \mathbf{b})=\mathbf{b} \cdot \nabla \times \mathbf{a}-\mathbf{a} \cdot \nabla \times \mathbf{b}$ have been used. In a 1-D anisotropic medium, both $\mathbf{H}^{(1)}$ and $\mathbf{H}^{(2)}$ are horizontal fields, and their vector product is $\mathbf{H}^{(1)} \times \mathbf{H}^{(2)}=D_{H} \mathbf{e}_{z}$, where $\mathbf{e}_{z}$ is a unit vector in the vertical direction. Then, Eq. (A.3) finally gives

$$
Z_{A}=D_{H}^{-1} D_{H}^{\prime}
$$

As $D_{H} \neq 0$ due to the independence of the two field polarization involved, Eq. (A.1) and the result (A.4) can be combined to

$$
S_{1}^{\prime} D_{H}+S_{1} D_{H}^{\prime}=\left(S_{1} D_{H}\right)^{\prime}=0 .
$$

Consequently, the product $S_{1} D_{H}$ is constant throughout the 1-D anisotropic medium. If $S_{1}=0$ at an arbitrary $z$ within the conductor, which is true, e.g., in the homogeneous basement of the model (see (17) for this particular case), then $S_{1}=Z_{x x}+Z_{y y}=0$ everywhere in the medium.

\section{References}

Abramovici, F., The forward magnetotelluric problem for an inhomogeneous and anisotropic structure, Geophysics, 39, 56-68, 1974.

Bahr, K., Geological noise in magnetotelluric data - a classification of distortion types, Phys. Earth Planet. Int., 66, 24-38, 1991.

Dekker, D. L. and L. M. Hastie, Magnetotelluric impedances of an anisotropic layered Earth model, Geophys. J. R. astr. Soc., 61, 11-20, 1980.

Dmitriev, V. I. and M. N. Berdichevsky, The fundamental model of magnetotelluric sounding, Proc. IEEE, 67, 1034-1044, 1979.

Groom, R. W. and R. C. Bailey, Decomposition of magnetotelluric impedance tensors in the presence of local 3-dimensional galvanic distortion, J. Geophys. Res.-Solid Earth and Planets, 94, 1913-1925, 1989.

Hindmarsh, A. C., ODEPACK, a systematized collection of ODE solvers, in Scientific Computing, edited by R. S. Stepleman et al., pp. 55-64, North-Holland, Amsterdam, 1983.

Jones, A. G. and R. W. Groom, Strike angle determination from the magnetotelluric impedance tensor in the presence of noise and local distortion: rotate at your peril!, Geophys. J. Int., 113, 524-534, 1993.

Loewenthal, D. and M. Landisman, Theory for magnetotelluric observations on the surface of a layered anisotropic halfspace, Geophys. J. R. astr. Soc., 35, 195-214, 1973.

O'Brien, D. P. and H. F. Morrison, Electromagnetic fields in an N-layered anisotropic half-space, Geophysics, 32, 668-677, 1967.

Petzold, L. R., Automatic selection of methods for solving stiff and nonstiff systems of ordinary differential equations, SIAM J. Sci. Stat. Comput., 4, 136-148, 1983.

Ralston, A., A First Course in Numerical Analysis, McGraw-Hill, New York, 578 pp., 1965.

Reddy, I. K. and D. Rankin, Magnetotelluric effect of dipping anisotropies, Geophys. Prosp., 19, 84-97, 1971

Santos, F. A. M. and L. A. Mendes-Victor, 1D anisotropic versus 2D isotropic media in magnetotellurics, Acta Geod. Geoph. Hung., 35, 49$61,2000$.

Singh, R. P. and Y. Kant, Sensivity analysis of EM measurements over exponential varying conductivity earth models, Geophys. J. Int., 121, 111-116, 1995.

Smith, J. T., Estimating galvanic distortion magnetic fields in magnetotellurics, Geophys. J. Int., 130, 65-72, 1997.

S. Kováčiková (e-mail: svk@ig.cas.cz) and J. Pek (e-mail: jpk@ig. cas.cz) 